\section{Agomelatine - is it another reboxetine? Another case of publication bias}

I read the special article about agomelatine with interest. ${ }^{1}$ The authors state that controlled studies have suggested a favourable efficacy and tolerability profile of agomelatine in depression. This statement is not entirely accurate.

The article has missed the negative studies and is a glaring example of publication bias, issues that have been highlighted in a recent meta-analysis. ${ }^{2}$ This meta-analysis of placebo-controlled trials of agomelatine in depression included unpublished trials and concluded that agomelatine is unlikely to be clinically superior to placebo. I am part of a group which has recently submitted a systematic review for the Cochrane Collaboration where we compared the efficacy of agomelatine with other antidepressant drugs in depression. Agomelatine did not seem to provide any significant advantage in efficacy. We also found evidence of publication bias. We contacted Servier, maker of agomelatine, for the unpublished trials, but did not receive any response. Furthermore, Servier has not provided data to the National Institute for Health and Care Excellence (NICE); hence NICE has not recommended agomelatine use. ${ }^{3}$

The article states that more data are needed to assess the effectiveness of agomelatine in real-world conditions. However, the fact is that agomelatine's efficacy in controlled trials is not yet established. Almost all the studies have been sponsored by Servier or Novartis, the company which marketed it in the USA.

Reboxetine is a classic example of publication bias; in this case mostly positive studies were published. Many years after its introduction, in 2010, the unpublished data was accessed and a meta-analysis found reboxetine to be an ineffective and potentially harmful antidepressant drug. ${ }^{4}$ It is time that drug companies disclose all data from all trials irrespective of the outcome so the efficacy of a drug can be judged objectively.

1 Whiting D, Cowen PJ. Drug information update: agomelatine. Psychiatrist 2013; 37: 356-8.

2 Koesters M, Guaiana G, Cipriani A, Becker T, Barbui C. Agomelatine efficacy and acceptability revisited: systematic review and metaanalysis of published and unpublished randomised trials. Br J Psychiatry 2013; 203: 179-87.

3 National Institute for Health and Clinical Excellence. Agomelatine for the Treatment of Major Depressive Episodes (Terminated Appraisal) (Technology Appraisal TA231). NICE, 2011.

4 Eyding D, Lelgemann M, Grouven U, Hrter M, Kromp M, Kaiser T, et al. Reboxetine for acute treatment of major depression: systematic review and meta-analysis of published and unpublished placebo and selective serotonin reuptake inhibitor controlled trials. BMJ 2010; 341: c4737.

Sumeet Gupta, Consultant Psychiatrist, Tees, Esk and Wear Valleys NHS Foundation Trust, Darlington, email: sumeet.gupta@nhs.net

doi: $10.1192 / \mathrm{pb} .38 .2 .88$

Authors' response: By taking part of a single sentence out of context, Sumeet Gupta misrepresents our article. We wrote: 'Controlled studies have suggested favourable efficacy and tolerability profiles; however, agomelatine is not without its controversies, with recent meta-analyses showing only marginal advantages over placebo' (our italics). We also stated that, 'although narrative reviews of the efficacy of agomelatine emphasise its superior efficacy relative to placebo and certain other antidepressants such as sertraline and fluoxetine, formal meta-analyses have found these effects to be less convincing and of uncertain clinical significance' (the meta-analysis by Koesters et al ${ }^{1}$ had not been published when we submitted our article, so we relied on that of Singh et $a l^{2}$ which reaches similar conclusions). Merely reading the abstract is enough to encounter the phrase: 'Current meta-analyses show marginal clinical benefits of agomelatine relative to placebo'. Overall, our conclusion is similar to that of Koesters et al': 'The present systematic review found that acute treatment with agomelatine is associated with a difference of 1.5 points on the HRSD. This difference was statistically significant, although the clinical relevance of this small effect is questionable'.

Drug companies are often accused, with justification, of making exaggerated and misleading claims. Their critics should avoid emulating them.

Declaration of interest: P.J.C. has been a paid member of advisory boards for Eli Lilly, Lundbeck and Servier.

1 Koesters M, Guaiana G, Cipriani A, Becker T, Barbui C. Agomelatine efficacy and acceptability revisited: systematic review and metaanalysis of published and unpublished randomised trials. Br J Psychiatry 2013; 203: 179-87.

2 Singh SP, Singh V, Kar N. Efficacy of agomelatine in major depressive disorder: meta-analysis and appraisal. Int J Neuropsychopharmacology 2012; 15: 417-28.

Philip J. Cowen, Professor of Psychopharmacology,

Daniel Whiting, Academic Clinical Fellow, University of Oxford, email: phil.cowen@psych.ox.ac.uk

doi: $10.1192 / p b .38 .2 .88 a$

\section{A leaflet to improve knowledge and attitudes to help-seeking for mental illness among Muslims}

Help-seeking for mental illness is problematic among Muslim communities in Western countries. ${ }^{1,2}$ We set out to develop a leaflet on attitudinal and knowledge barriers to help-seeking for mental illness in UK Muslims, working with a local voluntary organisation (SMART), the Royal College of Psychiatrists (RCPsych) and the Muslim Council of Britain. In the leaflet, we addressed known barriers to help-seeking such as cultural and traditional beliefs, knowledge of and familiarity with formal services, perceived societal stigma, and the use of informal indigenous resources. ${ }^{3}$ An Islamic religious leader checked the content for religious accuracy and we ensured the design was culturally consistent. A draft of the leaflet was piloted with a focus group of six Muslim men, then re-drafted using their feedback to produce the final version.

To evaluate the leaflet, we attended a London mosque at evening prayer time (Isha) and invited members of the congregation to complete a questionnaire before and after reading it. Twenty-five men aged $18-65+$ volunteered; there were no women at the mosque at the time. All were Muslim UK residents: 32\% Asian/Asian British Pakistani, 20\% Asian/ Asian British other, $44 \%$ other ethnicities combined, $4 \%$ gave no response. A statistically significant change was noted in response to two questions: 'I would see a doctor if I felt very sad, worried, scared or was having unusual experiences' $(P=0.039)$ and 'I know what treatments are available for mental illnesses' $(P=0.010)$. Furthermore, $72 \%$ of participants thought the leaflet helped them to understand mental illness better, $96 \%$ found it easy to read, $88 \%$ easy to understand and 